\title{
Management of Pod Bug, Clavigralla gibbosa Spinola (Heteroptera: Coreidae) on Pigeonpea
}

\author{
B.R. Chethan ${ }^{1}$, V. Rachappa $^{1 *}$, S.G. Hanchinal ${ }^{1}$, N.R. Harischandra ${ }^{1}$ and \\ S.R. Doddagoudar ${ }^{2}$ \\ ${ }^{1}$ Department of Agricultural Entomology, ${ }^{2}$ Department of Seed Science and Technology, \\ University of Agricultural Sciences, Raichur - 584104, Karnataka, India \\ *Corresponding author
}

\section{Keywords}

Clavigralla gibbosa, Insecticides,

Pigeonpea, Pod bug

\section{Article Info}

Accepted:

12 October 2018

Available Online:

10 November 2018

\section{A B S T R A C T}

The study was carried out to evaluate some insecticides against pod bug, Clavigralla gibbosa Spinola in pigeonpea during kharif 2017-18 at Agricultural Research Station, Kalaburagi, Karnataka, India under field conditions with nine treatments and three replications. The flonicamid $50 \mathrm{WG}$ recorded significantly lowest population of 0.67 bugs per plant while the bug population in acephate $75 \mathrm{SP}$ and thiamethoxam $25 \mathrm{WG}$ was 0.87 and $1.00 \mathrm{bug} / \mathrm{plant}$, respectively and all the three were statistically on par. The maximum population reduction over control was registered in the plot treated with flonicamid $50 \mathrm{WG}$ (94.91\%) followed by acephate 75 SP $(93.22 \%)$, tolfenpyrad 15 EC $(79.37 \%)$ and fipronil 5 EC $(77.97 \%)$ at 10 days after spray. Pod damage due to pod bug was lowest in plot treated with flonicamid $50 \mathrm{WG}(9.33 \%)$ followed by acephate $75 \mathrm{SP}(10.67 \%)$ and highest in buprofezin $20 \mathrm{SC}(16.33 \%)$. The grain damage was lowest in flonicamid $50 \mathrm{WG}$ $(6.77 \%)$ followed by acephate $75 \mathrm{SP}(8.72 \%)$. Highest grain yield of $1313.27 \mathrm{~kg} / \mathrm{ha}$ was registered in flonicamid $50 \mathrm{WG}$ followed by acephate $75 \mathrm{SP}(1270.31 \mathrm{~kg} / \mathrm{ha})$. Similarly, highest benefit: cost ratio of 2.42 in flonicamid $50 \mathrm{WG}$ and 2.38 in acephate $75 \mathrm{SP}$ and was higher than other treatments. Hence, these two insecticides can be suggested for effective management of pigeonpea pod bug.

\section{Introduction}

Pigeonpea [Cajanus cajan (L.) Millsp.] is the second most important pulse crop of the country next to chickpea. A number of factors are responsible for the lower productivity of pigeonpea of which mainly includes biotic as well as abiotic factors. Among biotic factors, insect pests are the key elements contributing for the economic loss of pigeonpea both in field and storage. A large number of insect pests (more than 300 species) attack pigeonpea (Prasad and Singh, 2004). Insects that attack the reproductive structures of plant cause maximum yield losses (Rangaiah and Sehgal, 1984). Among the pod damaging insect pests of pigeonpea next to pod borer, Helicoverpa armigera Hubner, Maruca vitrata 
(Fab.) and pod fly, Melanagromyza obtusa (Malloch) pod bug, Clavigralla gibbosa Spinola is most important pest in India inflicting heavy loss to seed yield. The pod bug damage recorded 25 to 40 per cent in pigeonpea and also can reduce pod and grain weight by 27 and 30 times respectively at 12 nymphs/plant (Adati et al., 2007). The economic threshold level of one nymph/plant and economic injury level of two nymphs/plant have potential to cause the yield loss.

In recent years pod bug, C. gibbosa causing threat to quality grain production in pigeonpea. Both nymphs and adults of the pod bug suck the sap from pods, grains, flowers and flower buds. The punctured pods exhibit reddish brown to dark brown patches on the surface of pods and premature drying of pods is noticed. When such pods are opened, shrivelled and malformed seeds are observed. Further such seeds are easily succumbed to secondary infection by fungal disease and pose problems in post harvesting processes. The main reason for outbreak of the pest is due to continuous and indiscriminate use of same insecticide, monocropping and introduction of early and extra early maturing pigeonpea genotypes (Bharathimeena and Sudharma, 2009; Hanumanthaswamy et al., 2009) and also due to favourable temperature and humidity during reproductive stage of the crop (Singh et al., 2008). Under these circumstances the investigation on pod bug particularly on management of this pest needs to be strengthened as the available in formation is very meagre. Keeping this point in view, present investigation was carried out for the management of pod bug, C. gibbosa in pigeonpea.

\section{Materials and Methods}

The present investigation was conducted at Agricultural Research Station, Kalaburagi during kharif 2017. The experiment was conducted in randomized block design (RBD) to measure the efficacy of different new chemistry insecticides. Nine chemical insecticides viz., buprofezin $20 \mathrm{SC}$, flonicamid $50 \mathrm{WG}$, tolfenpyrad $15 \mathrm{EC}$, fipronil $5 \mathrm{EC}$, thiamethoxam $25 \mathrm{WG}$, diafenthiuron $50 \mathrm{WP}$, dinotefuran $20 \mathrm{SG}$, acephate $75 \mathrm{SP}$ and untreated control (Table 1) where evaluated against $C$. gibbosa in the field conditions with three replications. The variety TS-3R was sown on $4^{\text {th }}$ July 2017 in a plot size of $6.3 \mathrm{~m} \mathrm{x}$ $4 \mathrm{~m}$ with a spacing of $90 \mathrm{~cm}$ between rows and $20 \mathrm{~cm}$ between plants were maintained.

All the package of practices (Anon. 2017) was followed, except for the management of sucking pests. Pod borer, Helicoverpa armigera was managed by manual collection and repeated spraying of $H a N P V$. The treatments were imposed twice with first application at tender pod stage followed by second spray after 20 days with Knapsack sprayer fitted with hollow cone nozzle. Observations were recorded at harvest for both pod and grain damage from five randomly selected plants from each plot. For pod damage all the pods of five selected plants were assessed. Hundred pods were selected randomly among the pods of five plants for estimating grain damage. The per cent pod damage, per cent grain damage and grain yield per plot were recorded. The grain yield $(\mathrm{kg}$ $\mathrm{ha}^{-1}$ ) was computed and subjected to statistical analysis.

\section{Results and Discussion}

Prior to imposition of treatments pest population count was made on one day before spray and it revealed that all the treatments including check had pod bug population ranging from 3.00 to 3.47 bugs per plant and were statistically non-significant indicating uniformity of pest population in the experimental plot (Table 1). Ten days after 
treatment, the effect of treatments was more prominent indicating real efficacy of chemicals. Bug population was ranged from 0.67 to 3.33 per plant. The flonicamid $50 \mathrm{WG}$ recorded lowest population of 0.67 bugs per plant while the bug population in acephate 75 SP and thiamethoxam $25 \mathrm{WG}$ was 0.87 and $1.00 \mathrm{bug} / \mathrm{plant}$, respectively and all the three were statistically on par (Table 1). The treatments like tolfenpyrad $15 \mathrm{EC}$, fipronil 5 $\mathrm{EC}$, dinotefuran $20 \mathrm{SG}$ and diafenthiuron 50 WP recorded a population of $1.07,1.13,1.33$ and 1.47 bugs per plant respectively and were on par with each other. The maximum bugs population (3.33 bugs/plant) was observed in untreated check. Reduction of pod bug population over control was worked out and found highest reduction in flonicamid $50 \mathrm{WG}$ (79.70 \%) followed by acephate $75 \mathrm{SP}$ and tolfenpyrad 15 EC $(73.87 \%)$. The least reduction of bug population among chemical treatments was seen in the buprofezin $20 \mathrm{SC}$ $(49.87 \%)$ sprayed plots (Table 1$)$.

Bug population one day before second spray ranged from 2.40 to 3.40 per plant (Table 1). The minimum number of bugs recorded on flonicamid 50 WG (2.40 bugs/plant) which was significantly superior over other treatments. Significantly highest bug population (3.40 bugs / plant) was observed in untreated check. Ten days after second spray, it was drastically decreased treated plots.

The effective molecule flonicamid 50 WG recorded lowest population of 0.15 bugs per plant while the bug population in acephate 75 SP (0.20 bug/plant) and were statistically at par. The treatments like tolfenpyrad $15 \mathrm{EC}$ and fipronil $5 \mathrm{EC}$ recorded a population of 0.61 and 0.65 bug per plant respectively and were on par with each other. Diafenthiuron 50 WP was the least effective treatment recorded 0.85 bug per plant and it was significantly superior over control (Table 1). The maximum population reduction over control was observed in the plot treated with flonicamid 50 WG $(94.91 \%)$ followed by acephate 75 SP (93.22\%), tolfenpyrad 15 EC (79.37\%) and fipronil $5 \mathrm{EC}$ (77.97 \%). Similarly, on Bt cotton Chandi et al., (2016) found that flonicamid $50 \mathrm{WG}$ was effective in managing the sucking insect pests particularly leafhoppers. Kumar and Nath (2003) demonstrated that insecticidal applications significantly controlled $C$. gibbosa on pigeonpea and all the treatments were found superior over the control.

The per cent pod damage ranged from 9.33 percent in flonicamid $50 \mathrm{WG}$ to $16.33 \%$ in buprofezin $20 \mathrm{SC}$. While in control plot the damage was $19.33 \%$. All the treatments were found to be superior over control with respect to percent pod damage. The relative performance of various insecticides on pod damage against pod bug was found in order of flonicamid $50 \mathrm{WG}(9.33 \%)>$ acephate $75 \mathrm{SP}$ $(10.67 \%)>$ tolfenpyrad 15 EC $(12.67 \%)>$ thiamethoxam 25 WG (13.00\%) > fipronil 5 EC $(13.33 \%)>$ diafenthiuron 50 WP (14.67\%) $>$ Dinotefuran 20 SG (15.00\%) > buprofezin 20 SC $(16.33 \%)$. Further untreated control recorded the highest pod damage of 19.33 per cent and was found to be significantly inferior to all other treatments (Table 2). The present findings on efficacy of acephate $75 \mathrm{SP}$ were in corroborates with Gopali et al., (2013) found that methomyl 40 SP @ 1.0g/l was superior followed by chlorpyriphos 20 EC @ $2.5 \mathrm{ml} / 1$ and acephate 75 SP @ 1.0g/l. They opined that broad-spectrum insecticides listed above were most effective in suppressing the pod bug population and recorded higher grain yield with maximum net profit and $\mathrm{B}$ : $\mathrm{C}$ ratio. The flonicamid $50 \mathrm{WG}$ reduced 51.72 per cent pod damage over untreated control followed by acephate $75 \mathrm{SP}$, tolfenpyrad $15 \mathrm{EC}$ and thiamethoxam $25 \mathrm{WG}$ were $44.83,34.48$ and 32.76 per cent, respectively (Table 1 ). The minimum pod damage reduction over control was observed in buprofezin 20 SC (15.52\%). 
Table.1 Effect of insecticides on the population of pod bugs

\begin{tabular}{|c|c|c|c|c|c|c|}
\hline \multirow[t]{3}{*}{ Treatments } & \multicolumn{6}{|c|}{ bugs per plant } \\
\hline & \multicolumn{3}{|c|}{ First spray } & \multicolumn{3}{|c|}{ Second spray } \\
\hline & $1 \mathrm{DBS}$ & 10 DAS & $\begin{array}{l}\text { Reduction } \\
\text { over } \\
\text { untreated } \\
\text { check }(\%)\end{array}$ & 1 DBS & $\begin{array}{c}10 \\
\text { DAS }\end{array}$ & $\begin{array}{c}\text { Reduction } \\
\text { over } \\
\text { untreated } \\
\text { check }(\%)\end{array}$ \\
\hline $\begin{array}{l}T_{1} \text { : Buprofezin } 20 \mathrm{SC} \\
\text { @ } 200 \text { g.a.i.//ha }\end{array}$ & $\begin{array}{c}3.40 \\
(1.97)^{\mathrm{a}}\end{array}$ & $\begin{array}{l}1.67 \\
(1.47)^{\mathrm{e}}\end{array}$ & 49.39 & $\begin{array}{l}3.20 \\
(1.92)^{\mathrm{de}}\end{array}$ & $\begin{array}{c}0.82 \\
(1.15)^{\mathrm{b}}\end{array}$ & 72.20 \\
\hline $\begin{array}{l}T_{2} \text { :Flonicamid } 50 \\
\text { WG @ } 100 \text { g.a.i./ha }\end{array}$ & $\begin{array}{c}3.20 \\
(1.92)^{\mathrm{a}}\end{array}$ & $\begin{array}{c}0.67 \\
(1.08)^{\mathrm{a}}\end{array}$ & 79.70 & $\begin{array}{c}2.40 \\
(1.70)^{\mathrm{a}}\end{array}$ & $\begin{array}{c}0.15 \\
(0.81)^{\mathrm{a}}\end{array}$ & 94.91 \\
\hline $\begin{array}{l}T_{3}: \text { Tolfenpyrad } 15 \\
\text { EC @ } 150 \text { g.a.i./ha }\end{array}$ & $\begin{array}{c}3.20 \\
(1.92)^{\mathrm{a}}\end{array}$ & $\begin{array}{l}1.07 \\
(1.25)^{\mathrm{bcd}}\end{array}$ & 67.87 & $\begin{array}{l}2.87 \\
(1.83)^{\mathrm{cd}}\end{array}$ & $\begin{array}{c}0.61 \\
(1.05)^{\mathrm{b}}\end{array}$ & 79.32 \\
\hline $\begin{array}{l}\mathrm{T}_{4} \text { :Fipronil } 5 \text { EC @ } \\
50 \text { g.a.i./ha }\end{array}$ & $\begin{array}{c}3.20 \\
(1.92)^{\mathrm{a}}\end{array}$ & $\begin{array}{c}1.13 \\
(1.27)^{\mathrm{bcd}}\end{array}$ & 66.07 & $\begin{array}{l}2.47 \\
(1.72)^{\mathrm{ab}}\end{array}$ & $\begin{array}{c}0.65 \\
(1.07)^{\mathrm{b}}\end{array}$ & 77.97 \\
\hline $\begin{array}{l}T_{5} \text { :Thiamethoxam } 25 \\
\text { WG @ } 50 \text { g.a.i./ha }\end{array}$ & $\begin{array}{c}3.47 \\
(1.99)^{\mathrm{a}}\end{array}$ & $\begin{array}{l}1.00 \\
(1.22)^{\mathrm{abc}}\end{array}$ & 69.97 & $\begin{array}{c}2.80 \\
(1.82)^{\mathrm{bcd}}\end{array}$ & $\begin{array}{c}0.71 \\
(1.10)^{\mathrm{b}}\end{array}$ & 75.93 \\
\hline $\begin{array}{l}\text { T}_{6} \text { :Diafenthiuron } 50 \\
\text { WP @ } 300 \text { g.a.i./ha }\end{array}$ & $\begin{array}{c}3.00 \\
(1.87)^{\mathrm{a}}\end{array}$ & $\begin{array}{l}1.47 \\
(1.40)^{\mathrm{de}}\end{array}$ & 55.86 & $\begin{array}{l}3.13 \\
(1.91)^{\mathrm{de}}\end{array}$ & $\begin{array}{c}0.85 \\
(1.16)^{b}\end{array}$ & 71.19 \\
\hline $\begin{array}{l}\text { T7:Dinotefuran } 20 \\
\text { SG @ } 40 \text { g.a.i.//ha }\end{array}$ & $\begin{array}{c}3.40 \\
(1.97)^{\mathrm{a}}\end{array}$ & $\begin{array}{l}1.33 \\
(1.35)^{\text {cde }}\end{array}$ & 60.06 & $\begin{array}{l}3.07 \\
(1.89)^{\mathrm{de}}\end{array}$ & $\begin{array}{c}0.79 \\
(1.14)^{\mathrm{b}}\end{array}$ & 73.22 \\
\hline $\begin{array}{l}\text { T: Acephate } 75 \text { SP @ } \\
750 \text { g.a.i./ha }\end{array}$ & $\begin{array}{c}3.33 \\
(1.96)^{\mathrm{a}}\end{array}$ & $\begin{array}{c}0.87 \\
(1.16)^{\mathrm{ab}}\end{array}$ & 73.87 & $\begin{array}{c}2.53 \\
(1.74)^{\mathrm{abc}}\end{array}$ & $\begin{array}{c}0.20 \\
(0.84)^{\mathrm{a}}\end{array}$ & 93.22 \\
\hline T9: Untreated control & $\begin{array}{c}3.40 \\
(1.97)^{\mathrm{a}}\end{array}$ & $\begin{array}{c}3.33 \\
(1.96)^{\mathrm{f}}\end{array}$ & - & $\begin{array}{c}3.40 \\
(1.97)^{\mathrm{e}}\end{array}$ & $\begin{array}{c}2.95 \\
(1.86)^{\mathrm{c}}\end{array}$ & - \\
\hline S.Em \pm & 0.04 & 0.05 & & 0.03 & 0.04 & \\
\hline $\mathrm{CD}$ at $(\mathrm{p}=0.05)$ & NS & 0.14 & & 0.10 & 0.12 & \\
\hline
\end{tabular}

Values in parenthesis are $\sqrt{\mathrm{x}}+0.5$ transformed

Means followed by same alphabet in columns did not differ significantly $(\mathrm{p}=0.05)$ by DMRT 
Table.2 Influence of insecticides spray on pod damage and yield of pigeonpea due to pod bug

\begin{tabular}{|c|c|c|c|c|c|c|}
\hline Treatments & $\begin{array}{c}\text { Pod } \\
\text { damage } \\
(\%)\end{array}$ & $\begin{array}{l}\text { Pod damage } \\
\text { reduction } \\
\text { over } \\
\text { control }(\%)\end{array}$ & $\begin{array}{l}\text { Grain } \\
\text { damage } \\
(\%)\end{array}$ & $\begin{array}{c}\text { Grain } \\
\text { damage } \\
\text { reduction } \\
\text { over } \\
\text { control } \\
(\%)\end{array}$ & $\begin{array}{l}\text { Grain } \\
\text { yield } \\
\text { (kg/ha) }\end{array}$ & $\begin{array}{c}\text { Increase } \\
\text { in yield } \\
\text { over } \\
\text { control } \\
(\%)\end{array}$ \\
\hline $\begin{array}{l}\mathrm{T}_{1} \text { : Buprofezin } 20 \mathrm{SC} \\
\text { @ } 200 \text { g.a.i./ha }\end{array}$ & $\begin{array}{c}16.33 \\
(23.82)^{\mathrm{e}}\end{array}$ & 15.52 & $\begin{array}{c}13.84 \\
(21.84)^{\mathrm{e}}\end{array}$ & 19.17 & $1098.18^{\mathrm{ef}}$ & 10.23 \\
\hline $\begin{array}{l}\mathrm{T}_{2} \text { :Flonicamid } 50 \mathrm{WG} \\
\text { @ } 100 \text { g.a.i./ha }\end{array}$ & $\begin{array}{c}9.33 \\
(17.78)^{\mathrm{a}}\end{array}$ & 51.72 & $\begin{array}{c}6.77 \\
(15.07)^{\mathrm{a}}\end{array}$ & 60.48 & $1313.27^{\mathrm{a}}$ & 31.81 \\
\hline $\begin{array}{l}\text { T}_{3}: \text { Tolfenpyrad } 15 \text { EC } \\
\text { @ } 150 \text { g.a.i./ha }\end{array}$ & $\begin{array}{c}12.67 \\
(20.83)^{b c}\end{array}$ & 34.48 & $\begin{array}{c}10.06 \\
(18.48)^{\mathrm{bc}}\end{array}$ & 41.25 & $1239.94^{\mathrm{abc}}$ & 24.45 \\
\hline $\begin{array}{l}\text { T4:Fipronil } 5 \text { EC @ } 50 \\
\text { g.a.i./ha }\end{array}$ & $\begin{array}{c}13.33 \\
(21.40)^{\mathrm{cde}}\end{array}$ & 31.03 & $\begin{array}{c}12.51 \\
(20.71)^{\text {de }}\end{array}$ & 26.96 & $1209.51^{\mathrm{bcd}}$ & 21.40 \\
\hline $\begin{array}{l}\text { T }_{5} \text { :Thiamethoxam } 25 \\
\text { WG @ } 50 \text { g.a.i./ha }\end{array}$ & $\begin{array}{c}13.00 \\
(21.11)^{\mathrm{cd}}\end{array}$ & 32.76 & $\begin{array}{c}11.46 \\
(19.77)^{\mathrm{cd}}\end{array}$ & 33.09 & $1154.27^{\text {cde }}$ & 15.86 \\
\hline $\begin{array}{l}\text { T6:Diafenthiuron } 50 \\
\text { WP @ } 300 \text { g.a.i./ha }\end{array}$ & $\begin{array}{c}14.67 \\
(22.50)^{\mathrm{cde}}\end{array}$ & 24.14 & $\begin{array}{c}12.78 \\
(20.93)^{\mathrm{de}}\end{array}$ & 25.38 & $1065.94^{\text {ef }}$ & 6.99 \\
\hline $\begin{array}{l}\text { T } 7 \text { :Dinotefuran } 20 \text { SG } \\
\text { @ } 40 \text { g.a.i./ha }\end{array}$ & $\begin{array}{c}15.00 \\
(22.78)^{\mathrm{de}}\end{array}$ & 22.41 & $\begin{array}{c}13.08 \\
(21.19)^{\mathrm{de}}\end{array}$ & 23.63 & $1136.65^{\mathrm{de}}$ & 14.09 \\
\hline $\begin{array}{l}\text { T8:Acephate } 75 \text { SP @ } \\
750 \text { g.a.i./ha }\end{array}$ & $\begin{array}{c}10.67 \\
(19.05)^{\mathrm{ab}}\end{array}$ & 44.83 & $\begin{array}{c}8.72 \\
(17.17)^{b}\end{array}$ & 49.06 & $1270.31^{\mathrm{ab}}$ & 27.50 \\
\hline $\mathrm{T}_{9}$ :Untreated control & $\begin{array}{c}19.33 \\
(26.08)^{f}\end{array}$ & & $\begin{array}{l}17.12 \\
(24.43)^{\mathrm{f}}\end{array}$ & & $996.30^{f}$ & \\
\hline S.Em \pm & 0.61 & & 0.48 & & 35.23 & \\
\hline CD at $(p=0.05)$ & 1.82 & & 1.45 & & 105.62 & \\
\hline
\end{tabular}

Values in parenthesis are arcsine transformed

Means followed by same alphabet in columns did not differ significantly $(\mathrm{p}=0.05)$ by DMRT 
Table.3 Cost economics of pod bug management through insecticides application

\begin{tabular}{|c|c|c|c|c|c|c|c|}
\hline Treatments & $\begin{array}{l}\text { Grain } \\
\text { yield } \\
\text { (kg/ha) }\end{array}$ & $\begin{array}{l}\text { Gross } \\
\text { returns } \\
\text { (Rs) }\end{array}$ & $\begin{array}{c}\text { Protection } \\
\text { cost (Rs) }\end{array}$ & $\begin{array}{l}\text { Crop } \\
\text { production } \\
\text { costs }(\mathrm{Rs})\end{array}$ & $\begin{array}{l}\text { Total } \\
\text { cost } \\
(\mathbf{R s})\end{array}$ & $\begin{array}{l}\text { Net } \\
\text { returns } \\
\text { (Rs) }\end{array}$ & $\begin{array}{l}\text { B:C } \\
\text { Ratio }\end{array}$ \\
\hline $\begin{array}{l}\mathrm{T}_{1} \text { : Buprofezin } 20 \\
\text { SC @ } 200 \text { g.a.i./ha }\end{array}$ & 1098 & 59841 & 2600 & 27170 & 29770 & 30071 & 2.01 \\
\hline $\begin{array}{l}T_{2} \text { :Flonicamid } 50 \\
\text { WG @ } 100 \text { g.a.i./ha }\end{array}$ & 1313 & 71559 & 2400 & 27170 & 29570 & 41989 & 2.42 \\
\hline $\begin{array}{l}T_{3}: \text { Tolfenpyrad } 15 \\
\text { EC @ } 150 \text { g.a.i.//ha }\end{array}$ & 1240 & 67580 & 8000 & 27170 & 35170 & 32410 & 1.92 \\
\hline $\begin{array}{l}\mathrm{T}_{4} \text { :Fipronil } 5 \text { EC @ } \\
50 \text { g.a.i./ha }\end{array}$ & 1210 & 65945 & 2800 & 27170 & 29970 & 35975 & 2.20 \\
\hline $\begin{array}{l}\text { T}_{5} \text { :Thiamethoxam } \\
25 \text { WG @ } 50 \\
\text { g.a.i./ha }\end{array}$ & 1154 & 62893 & 1480 & 27170 & 28650 & 34243 & 2.20 \\
\hline $\begin{array}{l}T_{6} \text { :Diafenthiuron } 50 \\
\text { WP @ } 300 \text { g.a.i./ha }\end{array}$ & 1066 & 58097 & 3920 & 27170 & 31090 & 27007 & 1.87 \\
\hline $\begin{array}{l}\text { T: }_{7} \text { :Dinotefuran } 20 \\
\text { SG @ } 40 \text { g.a.i./ha }\end{array}$ & 1137 & 61967 & 3200 & 27170 & 30370 & 31597 & 2.04 \\
\hline $\begin{array}{l}\text { T}_{8} \text { :Acephate } 75 \text { SP } \\
\text { @ } 750 \text { g.a.i./ha }\end{array}$ & 1270 & 69215 & 1920 & 27170 & 29090 & 40125 & 2.38 \\
\hline $\begin{array}{l}\text { Ty:Untreated } \\
\text { control }\end{array}$ & 996.3 & 54282 & - & 27170 & 27170 & 27112 & 2.00 \\
\hline $\begin{array}{c}\text { S.Em } \pm \\
\text { CD at }(p=0.05)\end{array}$ & & & & & & & \\
\hline
\end{tabular}

Market price of pigeonpea grains Rs. 5450 per quintal

$\mathrm{B}: \mathrm{C}$ ratio $=$ Gross returns $/$ Total cost

The treatments applied showed significant differences in the percent grain damage by pod bug and data are given in Table 2. The percent grain damage ranged from $6.77 \%$ in flonicamid $50 \mathrm{WG}$ to $13.84 \%$ in buprofezin $20 \mathrm{SC}$. While in control plot the damage was $17.12 \%$. All the treatments were found to be superior over control with respect to percent gain damage. The minimum grain damage was recorded in plot treated with flonicamid $50 \mathrm{WG}$ and it was significantly superior over all other treatments. The relative performance of various insecticides on grain damage against pod bug was found in order of flonicamid $50 \mathrm{WG}(6.77 \%)>$ acephate $75 \mathrm{SP}$ $(8.72 \%)>$ tolfenpyrad 15 EC $(10.06 \%)>$ thiamethoxam $25 \mathrm{WG}(11.46 \%)>$ fipronil 5
EC $(12.51 \%)>$ diafenthiuron 50 WP $(12.78 \%)>$ Dinotefuran 20 SG $(13.08 \%)>$ buprofezin 20 SC (13.84\%). Further untreated control recorded the highest grain damage of 17.12 per cent and was found to be significantly inferior to all other treatments. The flonicamid $50 \mathrm{WG}$ reduced 60.48 per cent grain damage over untreated control followed by acephate $75 \mathrm{SP}(49.06 \%)$ and tolfenpyrad 15 EC (41.25\%). The minimum grain damage reduction over control was observed in buprofezin 20 SC (19.17\%). Present findings were in line with Chandi et al., (2016) who found that Flonicamid $50 \mathrm{WG}$ was effective against sucking insect pests of Bt cotton. Similarly, Chinna et al., (2017) reported that flonicamid $50 \mathrm{WG}$ found most 
effective in controlling sucking pest population in cotton and also reported modern insecticides are relatively safer to the natural beneficial fauna. These reports support our findings on efficacy of Flonicamid 50 WG in bringing the population of pod bugs in pigeonpea.

The highest grain yield was recorded from flonicamid $50 \mathrm{WG}(1313.27 \mathrm{~kg} / \mathrm{ha})$ treated plot and minimum grain yield recorded from diafenthiuron 50 WP (1065.94 kg/ha) (Table 2). But all the insecticidal treatments recorded significantly higher grain yield as compared to untreated control $(996.30 \mathrm{~kg} / \mathrm{ha})$.

Further worked out the yield protection over untreated check and found that flonicamid 50 WG was superior $(31.81 \%$ more yield) followed by acephate 75 SP (27.50 \%), tolfenpyrad $15 \mathrm{EC}(24.45 \%)$ and fipronil 5 EC $(21.40 \%)$. The present result was in accordance with Namade et al., (2017) who stated flonicamid 50 WG @ $100 \mathrm{~g}$ a.i./ha was found very effective in controlling the cotton sucking pests and also gave higher yield.

The net profit was highest in flonicamid 50 WG (Rs. 41989/ha) followed by acephate 75 SP (Rs. 40125/ha) and fipronil 5 EC (Rs. 35975/ha). The lowest net profit was recorded in diafenthiuron $50 \mathrm{WP}$ (Rs. 27007/ha) and buprofezin 20 SC (Rs. 30071/ha), respectively (Table 3). Highest benefit: cost ratio was recorded in flonicamid 50 WG (2.42) followed by acephate $75 \mathrm{WS}$ (2.38).

Lowest benefit: cost ratio was recorded in diafenthiuron 50 WP (1.87) and tolfenpyrad 15 EC (1.92), respectively which were less than the untreated control (2.00).

The present study concluded that broad spectrum insecticides are effective in suppressing pod bug population with higher grain yield and $\mathrm{B}$ : $\mathrm{C}$ ratio like flonicamid 50
WG and acephate 75 SP against pod bug. Hence, these chemicals may be considered for recommendation in alternate sprays for managing the pod bug in pigeonpea.

\section{References}

Adati, T., Tamo, M., Yusuf, S. R., Downham, M. C. A., Singh, B. B. and Hammond, W., 2007, Integrated pest management for cowpea cereal cropping systems in the West African Savannah. Int. J. Trop. Insect Sci., 27(3/4): 123-37.

Anonymous, 2017, Package of Practices (for all regions of North Karnataka) University of Agricultural Sciences, Dharwad and University of Agricultural Sciences, Raichur, pp. 105-111.

Bharathimeena, T. and Sudharma, K., 2009, Preliminary efficacy of neem based insecticides against the pod bug Riptortus pedestris F. (Alydidae: Hemiptera). Insect Environ., 14(4): 161-162.

Chandi, R, S., Kumar, V., Bhullar, H. S. and Dhawan, A. K., 2016, Field efficacy of flonicamid 50 WG against sucking insect pests and predatory complex on Bt cotton. Indian J. Plant Prot., 44(1): 1-8.

Chinna, B. N., Kranthi, S. and Rahul V., 2017, Impact of newer pesticides and botanicals on sucking pest management in cotton under high density planting system (HDPS) in India. J. Entomol. Zool. Stud., 5(6): 1083-1087.

Gopali, J. B., Sharma, O. P., Yelshetty, S. and Rachappa, V., 2013, Effect of insecticides and biorationals against pod bug (Clavigralla gibbosa) in pigeonpea, Indian J. Agril. Sci., 83(5): 582-585.

Hanumanthaswamy, B. C., Yadahalli, K. B. and Nagaraja, M. V., 2009, Evaluation of bio intensive IPM module in redgram. Mysore J. Agril. Sci., 43(2): 386-388. 
Kumar, M. and Nath, P., 2003, Effect of insecticides on the extent of pod damage and seed damage by podfly, Melanagromyza obtusa (Malloch) and pod borer, Helicoverpa armigera (Hubner) in bahar cultivar of pigeonpea, Ann. Agril. Res., 24 (4): 934-942.

Nemade, P. W., Rathod, T. H., Deshmukh, S. B., Ujjainkar, V. V. and Deshmukh, V. V., 2017, Evaluation of new molecules against sucking pests of Bt cotton. $J$. Entomol. Zool. Stud., 5(6): 659-663.
Prasad, D. and Singh, A., 2004, Advances in Plant Protection Sciences. Akansha Publishing House, New Delhi: 421.

Rangaiah, P. V. and Sehgal, V. K., 1984, Insects on T-21 pigeonpea and losses caused by them at Pantnagar, Northern India. Int. Pigeonpea Newsletter, 3: 4043.

Singh, N. K., Anuradha, T. and Srivastava, O. P., 2008, Evaluation of certain newer insecticides against insect pest complex on pigeonpea (Cajanus cajan (L) Millsp.). J. Appl. Zool. Res., 19(1): 4649.

\section{How to cite this article:}

Chethan, B.R., V. Rachappa, S.G. Hanchinal, N.R. Harischandra and Doddagoudar, S.R. 2018. Management of Pod Bug, Clavigralla gibbosa Spinola (Heteroptera: Coreidae) on Pigeonpea. Int.J.Curr.Microbiol.App.Sci. 7(11): 1615-1622. doi: https://doi.org/10.20546/ijcmas.2018.711.183 